\title{
CULTIVANDO EL TALENTO MATEMÁTICO EN EDUCACIÓN INFANTIL MEDIANTE LA RESOLUCIÓN DE PROBLEMAS PARA FAVORECER EL DESARROLLO DEL PENSAMIENTO COMPUTACIONAL
}

\author{
Marta Terroba Acha \\ Juan Miguel Ribera Puchades \\ Daniel Lapresa Ajamil \\ Universidad de La Rioja
}

\begin{abstract}
RESUMEN: Por medio del empleo de un robot de suelo de direccionalidad programada, se ha trabajado una trayectoria de resolución de problemas para el desarrollo del pensamiento computacional. Se ha realizado un análisis de los procesos de pensamiento involucrados en la resolución de problemas cuya solución se puede representar mediante un conjunto ordenado de pasos. La puesta en marcha de este proyecto de programación y codificación en las aulas de $1^{\circ}, 2^{\circ}$ y $3^{\circ}$ de Educación Infantil, favorece el desarrollo del razonamiento matemático y de destrezas en la resolución de problemas en los escolares, al tiempo que permite desarrollar las habilidades de pensamiento computacional de los mismos. Los problemas propuestos han planteado retos desafiantes para todos los escolares en general y, para el alumnado de alta capacidad matemática en particular, proporcionando oportunidades únicas de razonamiento y de resolución de problemas. Los resultados obtenidos nos ofrecen información relevante relativa a la evolución de las habilidades del pensamiento computacional a lo largo de la etapa de Educación Infantil.
\end{abstract}

PALABRAS CLAVE: alta capacidad matemática, trayectoria de resolución de problemas, pensamiento computacional, robótica educativa, Educación Infantil.

\section{PROBLEM RESOLUTION TRAJECTORY FOR THE DEVELOPMENT OF COMPUTER THINKING. FOSTERING MATHEMATICAL TALENT IN EARLY CHILDHOOD EDUCATION}

\footnotetext{
ABSTRACT: Through the use of a programmed directionality floor robot, a problem-solving trajectory has been worked on for the development of computational thinking. An analysis of the thought processes involved in
} 
problem solving has been performed the solution of which can be represented by an orderly set of steps. The implementation of this programming and coding project in 1st, 2nd and 3rd Early Childhood Education classrooms, fosters mathematical reasoning development and skills in problem resolution in scholars, at the same time that develops the computational thinking skills in them. The proposed problems have set out challenging for all school children in general and, for high mathematical capacity students in particular, providing unique opportunities for reasoning and problem solving. The results obtained provide us with relevant information regarding the evolution of computational thinking skills throughout the Childhood Education Stage.

KEYWORDS: High mathematical capacity, problem solving trajectory, computational thinking, educational robotics, Early Childhood Education.

Recibido: 01/02/2021

Aceptado: 26/05/2021

Correspondencia: Marta Terroba Acha, Departamento de Ciencias de la Educación, Universidad de La Rioja, C/ Luis de Ulloa, 2, 26004. Logroño. Email: marta.terroba@ alum.unirioja.es

\section{INTRODUCCIÓN}

La introducción de la robótica educativa y el pensamiento computacional en las aulas, desde las primeras etapas educativas, está recibiendo un impulso a nivel internacional. Europa está asumiendo un papel activo al respecto y está promoviendo una agenda digital en la que la iniciación a la programación cobra vital importancia, asumida como la alfabetización de hoy en día (Bers et al., 2019; Manches y Plowman, 2017; Moreno-León y Robles, 2015).

La facilidad para acceder a los dispositivos tecnológicos desde edades tempranas y para integrarlos en todos los niveles educativos, permite introducir al alumnado en el lenguaje de la programación de un modo divertido en el que juego y aprendizaje van unidos (Bers, 2018). Entre las ventajas que origina la introducción de la programación en edades escolares cabe destacar el desarrollo de habilidades de resolución de problemas y de razonamiento matemático. Es por ello que la creación de ambientes tecnológicos de programación se está consolidando en el horizonte educativo (Benton et al., 2017; Clements y Samara, 2002; Hoyles y Lagrange, 2010; Leidl et al., 2017; Pérez y Diago, 2018; Shute et al., 2017; Sullivan y Bers, 2016).

Los proyectos de robótica que se trabajan en las aulas permiten a los estudiantes aprender a programar robots educativos o dispositivos tecnológicos que incluyen sistemas de codificación y se convierten en herramientas de primer orden para potenciar la adquisición de aprendizajes significativos (Terroba et al., 2020). El alumnado debe ser capaz de abstraer el modo de actuación que seguirá el objeto programable, funda- 
mentándose en el valor de los códigos incrustados y el efecto que produce su puesta en funcionamiento, desarrollando las destrezas del pensamiento computacional (Lee et al., 2011; Wing, 2006). Desde la primera conceptualización de pensamiento computacional acuñada por Wing (2006), en la que lo define diciendo que el pensamiento computacional "implica la resolución de problemas, el diseño de sistemas y, la comprensión de la conducta humana, haciendo uso de los conceptos fundamentales de la informática" (p. 33), ha habido muchas otras interpretaciones del término, no llegando a un acuerdo sobre una única formulación del mismo (Gouws et al., 2013; RománGonzález et al., 2015). El pensamiento computacional como medio de resolución de problemas conlleva el empleo de estrategias que el alumnado debe emplear para enfrentarse al problema con la limitación de que tiene que poder ponerse en funcionamiento en entornos tecnológicos (Diago et al., 2018a). Para desarrollar el pensamiento computacional en las aulas no es imprescindible el empleo de ordenadores; elementos tan simples como un lápiz y una hoja pueden ser recursos a emplear para lograrlo (Ribera, 2021). Al codificar los escolares aprenden a desarrollar un plan de acción, identifican patrones de repetición y descubren los fallos en su pensamiento computacional al descubrir que el programa desarrollado no funciona del modo esperado (Diago et al., 2018b; Valverde et al., 2015). El enorme potencial que supone trabajar el pensamiento computacional en las aulas puede relacionarse estrechamente con las fases establecidas por Pólya (1945) en la resolución de problemas: entender el problema, establecer un plan de actuación, ejecutar el plan y evaluar el proceso, puesto que el alumnado debe comenzar con la elaboración de un plan previo de actuación y puede evaluar el plan establecido en función de la respuesta del dispositivo tecnológico (Diago et al., 2018b).

La presente experimentación desarrolla por medio del pensamiento computacional una trayectoria de resolución de problemas matemáticos, contextualizados en un cuento, que debe implementarse con un robot de suelo de direccionalidad programada.

La enseñanza de materias como la robótica o la programación en esta primera etapa educativa en niños de Educación Infantil permiten, entre otros aspectos, desarrollar las habilidades de pensamiento computacional (Bers, 2008). Estudios realizados por diversos autores, señalan cómo las tareas propias de las ciencias de la computación mejoran el desarrollo del razonamiento matemático en los escolares y las destrezas en la resolución de problemas (Clements y Samara, 2002; Hoyles y Lagrange, 2010; Pérez y Diago, 2018). La resolución de problemas se constituye como un indicador del alumnado con alta capacidad matemática. Un elevado porcentaje de expertos centrados en la investigación de la superdotación en matemáticas coinciden en que la resolución de problemas matemáticos es una vía fundamental para caracterizar al alumnado con talento matemático (Castro et al., 2006). Las respuestas dadas a los problemas planteados, permiten detectar a los escolares con talento matemático si se tienen en consideración aspectos como la creatividad en las respuestas dadas o que dichas resoluciones sean de un nivel superior en función de la edad (Butto et al., 2016; Castro et al., 2006). El papel del maestro es fundamental para estimular en el alumnado el deseo de descubrir lo que no sabe y de impulsar acciones que fomenten la creatividad en sus respuestas (Taylor, 1964). Los escolares con talento matemático manifiestan más curiosidad por los retos más complejos, encuentran el quid de la cuestión fácilmente, las respuestas proporcionadas suelen ser originales y no se rinden con facilidad ante la dificultad de los problemas planteados (Freiman 2006). 
Los problemas que conforman la trayectoria de resolución de problemas que constituye la experimentación precisan que el alumnado emplee la inteligencia lógica para resolver los problemas, para lo cual resulta esencial su capacidad de razonamiento, de deducción y de abstracción (Etchepare et al., 2011). El contenido de los retos que se presentan requiere de la orientación espacial y lógica de los escolares. Los alumnos con talento matemático poseen una elevada capacidad de planificación, revisión y evaluación de sus actividades intelectuales que constituyen la denominada inteligencia lógica, lo que les habilita para un obtener un mayor rendimiento en las actividades matemáticas desde edades muy tempranas (Ferrándiz et al., 2010; Onrubia et al., 2003; Piñeiro et al., 2018).

Dentro de la trayectoria de la resolución de problemas que se expone, se proponen problemas abiertos, que no tienen una única solución, lo que además de constituirse como un desafío puede permitir desarrollar el potencial del alumnado con alta capacidad. Como indica Johnson (2000), por medio del análisis de los razonamientos que ofrece el alumnado se puede determinar la calidad de su pensamiento y por ende determinar si es talentoso en matemáticas. Las respuestas creativas o respuestas únicas pueden ser indicadores del estudiantado superdotado (Pitta-Pantazi, et al., 2011). La mente matemática de estos estudiantes les permite crear maneras originales para resolver problemas matemáticos (Krutetskii, 1976; Özdemir e Işiksal, 2019).

Greenes (1981) identifica una serie de características para determinar el talento matemático en la resolución de problemas. La resolución de los problemas que componen la propuesta desarrollada requiere de destrezas estrechamente relacionadas con dichas características. La flexibilidad en el manejo de datos queda patente en las diversas estrategias y enfoques utilizados por los escolares para dar respuesta a los problemas de solución abierta, más avanzados en la trayectoria. La capacidad de organización de datos se relaciona con la capacidad del alumnado para organizar la secuencia de instrucciones que servirá para recorrer el camino planificado. La agilidad mental de fluidez de ideas se refleja en los retos con obstáculos que pueden mostrar asociaciones únicas en las respuestas dadas. La originalidad de interpretación se ve en el problema cuya solución es que no hay solución posible. La capacidad de transferir ideas permite comprobar cómo los escolares son capaces de aplicar la información aprendida en los primeros problemas de la trayectoria a los retos de mayor dificultad a medida que avanza la propuesta. La habilidad para generalizar y establecer relaciones se manifiesta explícitamente en lo estudiantes altamente dotados. Los problemas planteados se incluyen dentro de lo que Greenes (1981) denomina problemas de lógica recreativa, que requieren el empleo de la lógica deductiva y se caracterizan por tener varios condicionantes para poder ser resueltos.

Los objetivos que se pretenden alcanzar con el desarrollo de la propuesta de desarrollo del pensamiento computacional mediante una trayectoria de resolución de problemas matemáticos son: a) Presentar una propuesta de trayectoria de resolución de problemas para el desarrollo del pensamiento computacional mediante el empleo de un robot de suelo de direccionalidad programada. b) Analizar la adecuación de la propuesta de resolución de problemas al pensamiento computacional del alumnado de los tres cursos del segundo ciclo de Educación Infantil, a partir de las resoluciones ofrecidas y del tiempo empleado en la resolución de cada uno de los problemas que componen la trayectoria. 


\section{Método}

La experiencia que se detalla se ha realizado en el CEIP La Guindalera, colegio de Educación Infantil y Primaria del municipio de Logroño, La Rioja, durante el curso escolar 2019/2020.

\subsection{Participantes}

Han participado en el estudio 71 alumnos/as distribuidos de la siguiente manera: 25 alumnos/as de $1^{\circ}$ de Educación Infantil, 22 alumnos/as de $2^{\circ}$ de Educación Infantil y 24 alumnos/as de $3^{\circ}$ de Educación Infantil.

El ensayo realizado consta de dos fases diferenciadas; una primera fase de activación, dirigida a todos los escolares de los grupos-clase, con el propósito de seleccionar la muestra y, una segunda fase, de ejecución de la trayectoria de resolución de problemas, realizada por el alumnado que ha superado la primera fase.

Todos los alumnos matriculados en los cursos de Educación Infantil han intervenido en una primera fase de selección de la muestra. No se ha realizado ningún estudio previo de talento matemático a este alumnado. Para realizar esta selección de la muestra se programaron una serie de tareas denominadas de activación, cuyo proceso de ejecución seguía dos partes diferenciadas: una primera, en la que el alumnado debía seleccionar las tarjetas adecuadas para realizar el recorrido y, una segunda, en la que los escolares tenían que realizar motrizmente el recorrido que indican las tarjetas. En la figura 1 se muestra cómo un alumno de $1^{\circ}$ de Educación Infantil realiza la tarea 1 de activación.

Figura 1. Tarea de activación 1

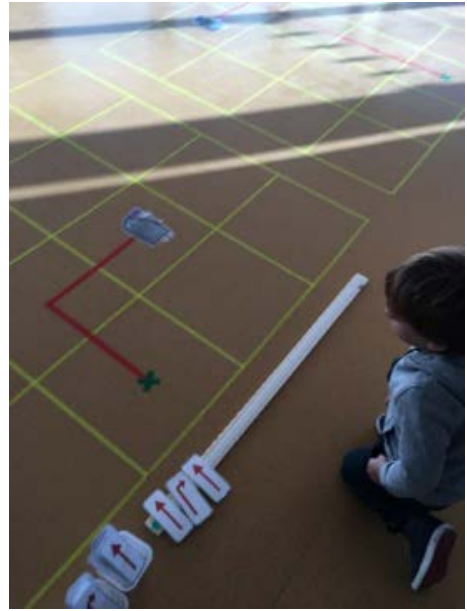

Fase de selección de tarjetas

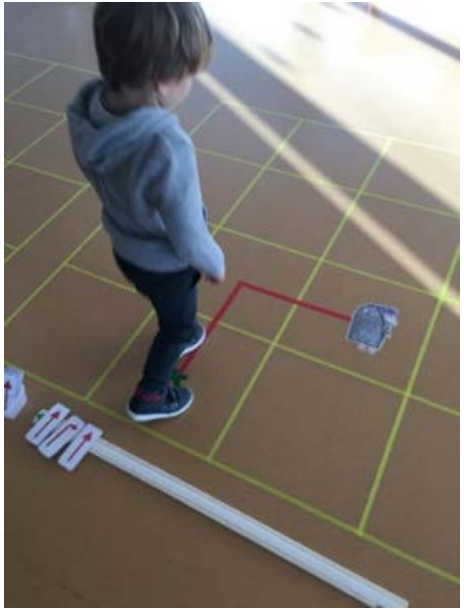

Fase de ejecución motriz

Dichas actividades se ejecutaron motrizmente en la sala de motricidad del centro educativo. Las tareas de activación se componen de varios ejercicios descontextuali- 
zados del cuento, enmarcados dentro de una trayectoria de resolución de problemas de dificultad creciente, cuya resolución requiere descubrir los pasos necesarios para conseguir llegar por el camino marcado hasta el punto señalado. En el suelo de la sala se dibujaron con cinta aislante, cinco cuadrículas con los cinco recorridos a realizar para superar esta primera fase del estudio. El primer recorrido era un camino recto, el segundo trayecto era un camino corto con un único giro, el tercero era un camino largo con sólo un giro, el cuarto era un camino con doble giro y el último un camino largo con doble giro y orientación opuesta. Atendiendo al desarrollo evolutivo del alumnado en relación con la orientación espacial, las tareas se distribuyeron para cada uno de los cursos. Las tres primeras tareas las ejecutaron los escolares de $1^{\circ}$ de Educación Infantil; las cuatro primeras las realizaron los estudiantes de $2^{\circ}$ de Educación Infantil; y, las cinco tareas, al completo, las efectuó el alumnado de $3^{\circ}$ de Educación Infantil (véase tabla 1).

Tabla 1. Tareas de activación para la selección de la muestra

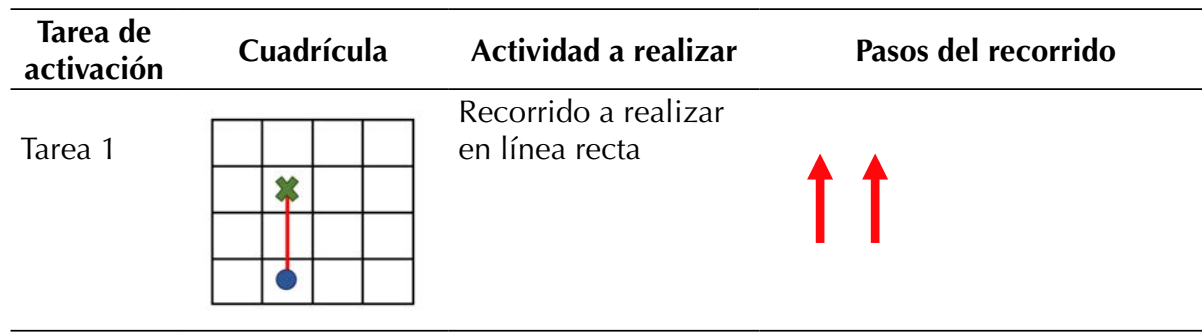

Tarea 2

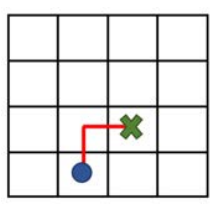

Recorrido corto a

realizar con un giro

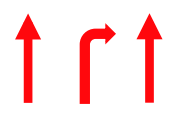

Recorrido largo a

Tarea 3

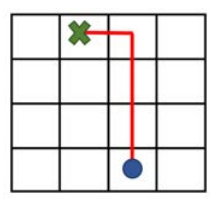

realizar con un giro

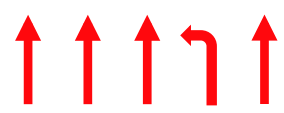

Tarea 4

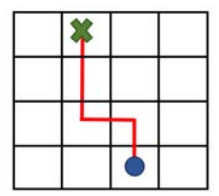

Recorrido largo a

realizar con dos giros

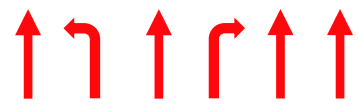

Tarea 5

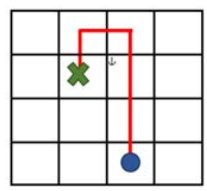

Recorrido largo a

realizar con dos

giros y orientación

espacial opuesta

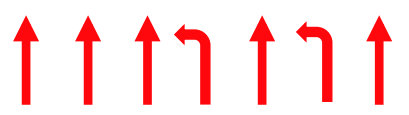


De los 71 participantes que realizaron esta fase de selección de la muestra, 34 escolares realizaron correctamente todas las tareas correspondientes a su nivel educativo: 12 de $1^{\circ}$ de Educación Infantil (con una media de edad de 3,72 años y una desviación típica de 0,23), 11 de $2^{\circ}$ de Educación Infantil (con una media de edad de 4,69 años y una desviación típica de 0,26) y 11 de $3^{\circ}$ de Educación Infantil (con una media de edad de 5,62 años y una desviación típica de 0,30) y fueron los que realizaron la trayectoria de resolución de problemas.

Para superar cada una de las tareas era preciso cumplir dos requisitos, que serán también condicionantes determinantes en la fase de experimentación; realizarlo en un número de intentos igual o inferior a tres y que el tiempo total de resolución no superara los cinco minutos. Estos condicionantes se sustentan en la capacidad de atención de los escolares de la etapa de Educación Infantil que va progresando con el transcurso del desarrollo cognitivo de los estudiantes. El tiempo medio de atención para el alumnado de esta etapa, no se recomienda que se alargue más de 30 minutos. La trayectoria se compone de siete problemas, se ha calculado un tiempo máximo de 5 minutos por problema, o un número límite de tres intentos, para que el proceso de resolución total se realice en un tiempo aproximado de media hora.

\subsection{Infraestructura y materiales}

Cada una de las sesiones que se llevaron a cabo en el aula de desdoble de Educación Infantil del CEIP La Guindalera de Logroño.

\section{Cuento "Chivos chivones"}

El cuento de la editorial Kalandraka "Chivos chivones" ha sido el eje motivador sobre el que ha girado todo el estudio. Previo a las sesiones de resolución de problemas la investigadora ha narrado el cuento a los escolares, con el fin de captar el interés del alumnado por resolver los retos planteados. La inclusión de este cuento para formular los problemas de la trayectoria de resolución de problemas ha permitido trasladar a los escolares a un mundo mágico, con personajes fantásticos, en los que se hacía necesaria su ayuda para resolver los retos planteados, cada vez más complicados de ejecutar. Todos los problemas han tenido un objetivo común, salvar a los chivos del ogro malvado que vivía sobre el puente, siguiendo el camino marcado o las indicaciones dadas por la maestra para su resolución.

\section{Robot Next 1.0.}

Next 1.0 es un robot de suelo que cuenta con programación direccional en la parte superior. Presenta cuatro comandos de movimientos disponibles: arriba, abajo, derecha e izquierda. También tiene comandos para pausar, borrar y/o comenzar a realizar la secuencia programada (véase tabla 2 ). 
Tabla 2. Comandos e instrucciones

\begin{tabular}{|c|c|}
\hline Comando & Instrucción \\
\hline & Flecha de dirección hacia adelante, el robot Next 1.0 avanza 14 centímetros \\
\hline & Flecha de dirección hacia atrás, hace que el robot retroceda 14 centímetros \\
\hline & $\begin{array}{l}\text { Flecha de giro a la derecha, hace que el robot gire } 90 \text { grados hacia la derecha, } \\
\text { pero no efectúa ningún desplazamiento }\end{array}$ \\
\hline & $\begin{array}{l}\text { Flecha de giro a la izquierda, hace que Next } 1.0 \text { gire } 90 \text { grados hacia la izquierda, } \\
\text { sin efectuar ningún desplazamiento }\end{array}$ \\
\hline & Comienzo de la programación \\
\hline & Pausado del recorrido \\
\hline
\end{tabular}

\section{Tarjetas de dirección}

Para llevar a cabo la programación del robot, se han utilizado tarjetas representativas de los movimientos que es capaz de ejecutar. Los movimientos del robot pueden ser de dos tipos, con desplazamiento y sin desplazamiento. Puede moverse un paso hacia delante y un paso hacia atrás, realizando una trayectoria equivalente a la medida de un cuadrado de las cuadrículas que se describen en el siguiente apartado. Puede realizar giros de $90^{\circ} \mathrm{sin}$ desplazamiento hacia la izquierda o hacia la derecha. Cada vez que se presiona una tecla en el dispositivo, el robot ejecuta un movimiento. Previo a la introducción de los códigos seleccionados en el robot, los escolares deben idear un plan de acción que ejecute el recorrido correcto para lo cual disponen de tarjetas de dirección. Los alumnos han tenido a su disposición un número suficiente de tarjetas para poder secuenciar el recorrido. Las tarjetas de instrucciones preparadas para la secuencia, son las señaladas en la tabla 3.

Tabla 3. Tarjetas de dirección

\begin{tabular}{ll}
\hline Tarjeta & Instrucción \\
& $\begin{array}{l}\text { Tarjeta de dirección hacia adelante } \\
\text { Tarjeta de dirección de giro hacia la }\end{array}$ \\
& $\begin{array}{l}\text { Tarjeta de dirección de giro hacia la la hacia atrás } \\
\text { izquierda }\end{array}$
\end{tabular}




\section{Tablero con cuadrícula blanca}

El tablero de cuadrícula blanca tiene un área total de $3136 \mathrm{~cm}^{2}$. Está formado por 16 cuadros de $196 \mathrm{~cm}^{2}$ cada uno. Cada uno de los cuadrados tiene la misma longitud de lado que el recorrido que realiza el robot cuando se selecciona un desplazamiento.

Sobre el tapete se marcarán diferentes caminos para que Next realice los recorridos. Serán dos los retos que deberán realizar los alumnos en la trayectoria de resolución de problemas, de dificultad creciente. Un primer reto, con un único giro. Un segundo problema, con dos giros.

Tablero con cuadrícula sobre paisaje de montañas

El tablero con cuadrícula sobre paisaje de montañas tiene las mismas dimensiones que el tapete de cuadrícula blanca.

El paisaje dibujado, detallado más adelante en la tabla 4, representa un paisaje del cuento sobre el que gira la resolución de problemas matemáticos "Chivos chivones".

\section{Muñequeras}

Para la resolución de los problemas, se han colocado muñequeras de dos colores al alumnado, verde para la mano derecha y roja para la mano izquierda, con el propósito de ayudar a los escolares con su orientación espacial.

\section{Aula}

Todas las actividades se han desarrollado en el aula de apoyo de Educación Infantil, que cuenta con espacio suficiente tanto para la sesión psicomotora, como para las sesiones de robótica.

\subsection{Procedimiento}

La investigación desarrolla una trayectoria de resolución de problemas de dificultad creciente para el desarrollo del pensamiento computacional mediante la utilización de un robot de suelo de direccionalidad programada.

Simon (1995) delimitó el término trayectoria de aprendizaje como un recorrido posible a través de la cual los estudiantes logran avanzar en su aprendizaje. Para configurarla es preciso tener en cuenta tres aspectos fundamentales: el objetivo planteado de aprendizaje, el proceso didáctico de desarrollo detallado y las actividades de enseñanza. Los objetivos planteados han quedado precisados en la introducción del artículo. Tanto el proceso didáctico como las actividades de enseñanza son los aspectos que van a reseñarse a continuación.

Previo a la trayectoria de resolución de problemas, se realizaron diversas actividades con el alumnado con el propósito de que aprendieran los códigos de las órdenes que se emplearían tanto en las tareas de activación como en la secuencia de resolución de problemas.

En un primer momento, en la sala de motricidad del centro, se explicaron los cuatro movimientos básicos del procedimiento: pasos hacia delante y hacia atrás con 
desplazamiento y giros hacia la derecha y hacia la izquierda sin desplazamiento. Se diseñaron unas tarjetas con las cuatro flechas de dirección y se ejecutaron juegos diversos en los cuales había que realizar los movimientos seleccionados. Atendiendo a la orden indicada por la maestra, el alumnado debía ejecutar los movimientos indicados, teniendo en cuenta si dicha consigna implicaba desplazamiento o no. Una vez que se tuvo la certeza de que el alumnado comprendía la diferencia entre giro y desplazamiento se dio por finalizada esta fase de preparación.

Tras la primera fase de selección de la muestra, se pasó a la fase de resolución de problemas englobados en una trayectoria de resolución, compuesta por ocho problemas, un primero con resolución motriz y los siete restantes para resolver utilizando el robot Next 1.0. Los cuatro primeros tienen una solución única, los tres siguientes una solución óptima y el último de ellos es un problema cuya solución es descubrir que no existe un recorrido viable. Por solución única se entiende aquella cuya respuesta ofrecida por los escolares se ajusta a los pasos estrictamente necesarios para recorrer el camino marcado sin salirse del trayecto. La solución óptima se refiere a aquellas respuestas que el alumnado da cumpliendo con los condicionantes marcados en el enunciado del problema, teniendo en cuenta que ésta puede ser diversa. El problema octavo de la trayectoria presenta una encrucijada sin recorrido posible y los estudiantes deben darse cuenta de que no es posible llegar hasta donde se les pide. Las respuestas ofrecidas por el alumnado pueden ser indicadores del talento matemático. Reyes-Santander y Karg (2009), señalan las características que posibilitan una aproximación a las características de los alumnos altamente dotados para las matemáticas, que son susceptibles de valoración en una clase de matemáticas. Dichas cualidades se pueden enmarcar dentro de las siguientes: despunte en el ámbito de la competencia matemática, tenacidad y perseverancia en la ejecución de actividades que les interesan, originalidad en las respuestas o productos y capacidad para generar ideas innovadoras, vanguardistas y abstractas en matemáticas, rendimiento e involucración en las tareas, capacidad de captación y comprensión de ideas complejas y manipulación de la información matemática. La estructura dada a la trayectoria descrita parte del problema motriz como problema uno, puesto que se considera más sencillo en los niños de estas edades partir de su propio cuerpo para resolver problemas de orientación espacial. El problema 2, se posiciona en este lugar puesto que se ha estimado que es el ejercicio más simple para realizar con el robot, debido a que implica un único giro sobre un camino pautado. El problema 3, continúa la trayectoria al ser un problema marcado en la cuadrícula con doble giro. El problema 4, añade la dificultad de no tener el camino marcado, aunque sí obstáculos que indican el camino permitido, supone un nivel más de complejidad dentro del doble giro. Los cuatro últimos problemas de la trayectoria, van incrementando la dificultad del recorrido a realizar y ofrecen diversas soluciones que pueden dan lugar a respuestas creativas y originales e incluso pueden ser respuestas que se encuentren a un nivel más elevado del que les corresponde por edad y ser por tanto indicadores de estudiantes con altas capacidades matemáticas (Castro et al., 2006).

Los problemas, los caminos y la secuencia de resolución se pueden observar en la tabla 4, que refleja la trayectoria de resolución de problemas planificada. 
Tabla 4. Trayectoria de resolución de problemas

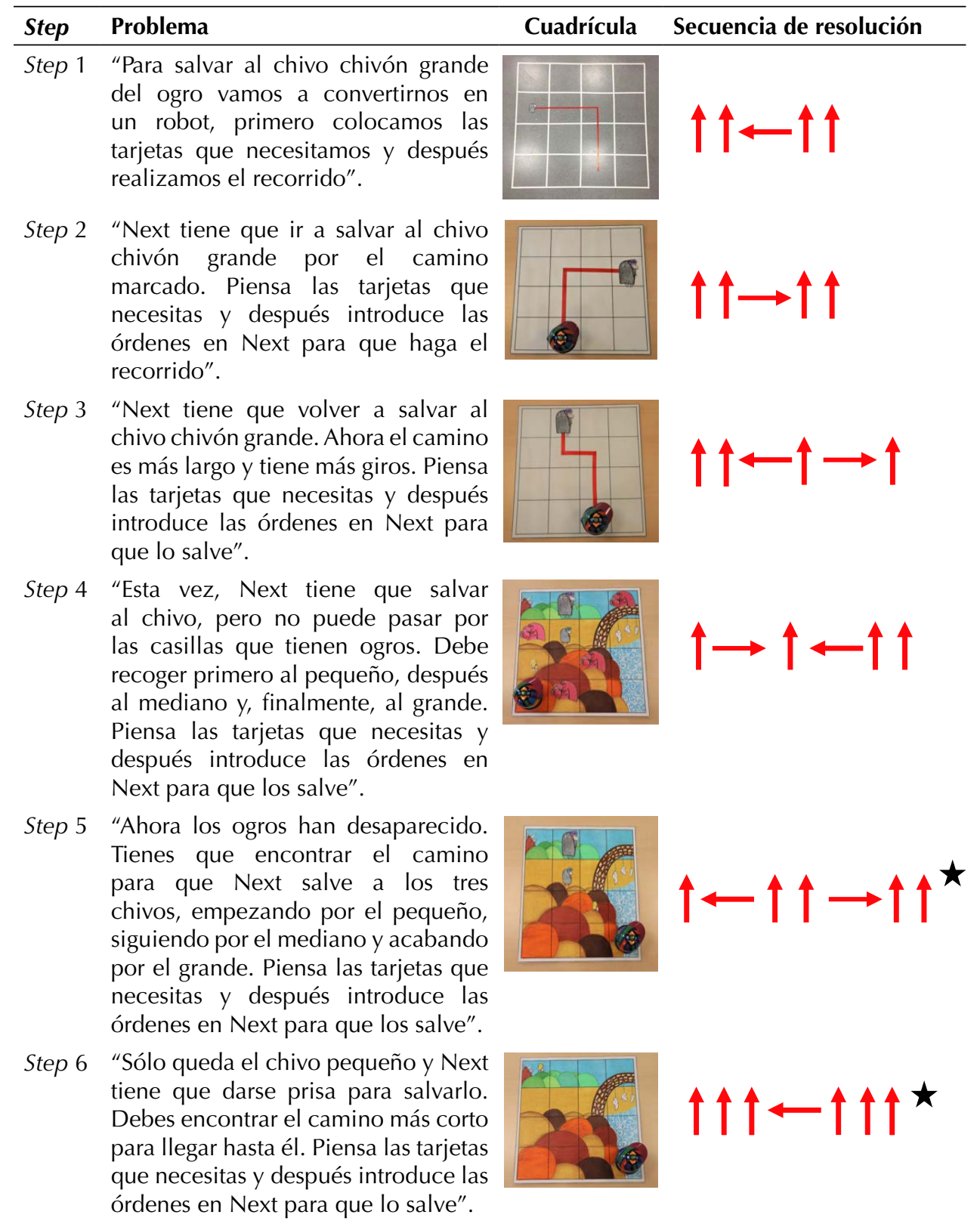




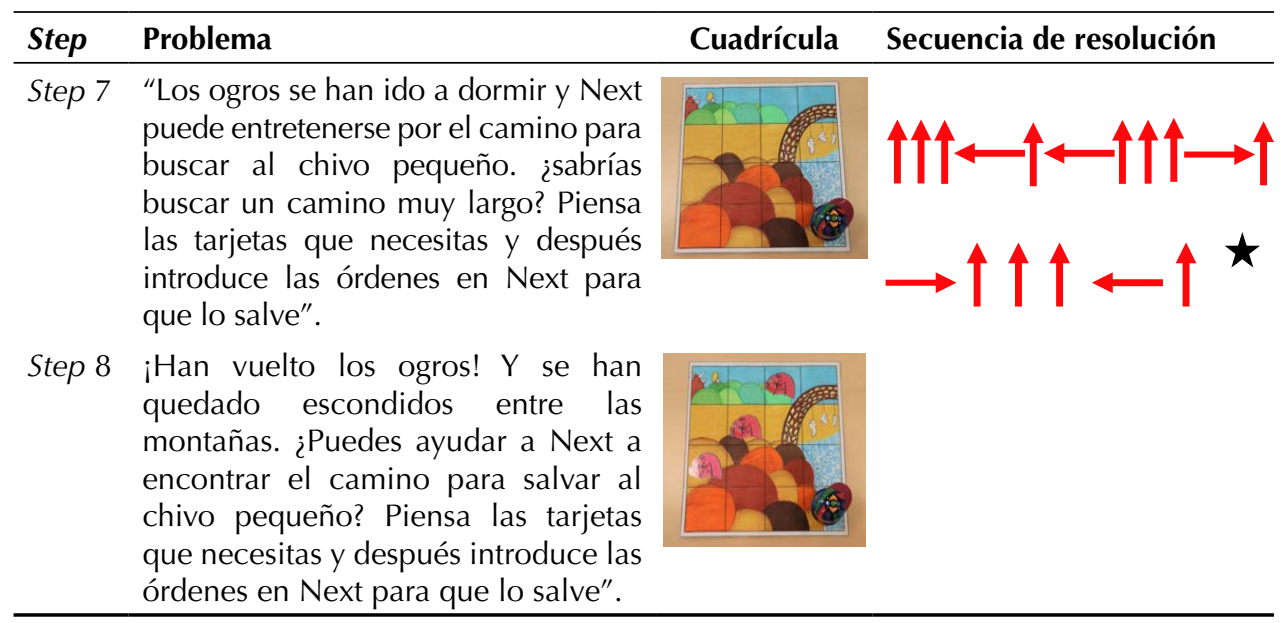

Problemas con solución abierta, se muestra un ejemplo de secuencia de resolución.

Durante la primera sesión de la trayectoria de resolución de problemas se explicó el funcionamiento de Next 1.0, los movimientos, los comandos y las tarjetas de dirección, con el propósito de dar la oportunidad al alumnado de manipular el robot y familiarizarse con su uso (Skoumpourdi, 2010). Aunque el dispositivo es bastante sencillo en cuanto a su uso, como indica Szendrei (1996), es primordial que los escolares aprendan su manejo correctamente.

Cada uno de los problemas de la trayectoria precisa, como mínimo, dos pasos para ser resueltos: la elección de las tarjetas del recorrido y la introducción en Next de los códigos seleccionados. En los problemas de solución óptima, también se añade un primer paso consistente en la determinación previa del recorrido que va a hacer el robot con el dedo.

Cada problema debe ser resuelto en un máximo de tres intentos o en un tiempo no superior a cinco minutos. Un intento termina cuando Next Ilega al punto final de su trayectoria. La eficacia de los intentos depende de su resolución, si Next consigue llegar hasta el chivo chivón siguiendo las condiciones descritas en el enunciado del problema se considera que es resuelto y se da paso al siguiente problema de la trayectoria. Si no se resuelve, debido a que se supera el número de intentos permitidos o el tiempo establecido para la resolución, los escolares pasan al siguiente problema y el anterior queda sin resolver.

La orientación de Next respecto a la posición de partida va variando a lo largo de la resolución. A medida que Next se va desplazando por las cuadrículas puede tener igual orientación que al principio, orientación opuesta o en espejo y orientación lateral, tanto derecha como izquierda. Lo mismo sucede con la posición de Next respecto a la posición del niño. En ambos casos, la necesidad de tener en consideración diversas referencias espaciales a la vez, supone una dificultad para el razonamiento de los escolares al precisar un descentramiento para lograr una resolución correcta al problema (Denis, 2017). 
A lo largo de las resoluciones, los estudiantes pueden retirar tarjetas de la secuencia que no conllevan a una respuesta adaptada a la solución posible. Puede ser retirada cualquier tarjeta, correcta, incorrecta, una serie de tarjetas hasta el primer error, la última tarjeta colocada o incluso todas las seleccionadas.

\subsection{Intervención de la maestra}

La intervención de la maestra se hace fundamental para potenciar el aprendizaje a través de un proceso educativo eminentemente lúdico, las interacciones entre maestra-alumno y alumno-Next desencadenan situaciones creativas de resolución de problemas en el alumnado (Kewalramani et al., 2020). Además, los docentes deben saber motivar e incentivar al alumnado para conseguir resolver los desafíos de aprendizaje (Pöntinen y Räty-Záborszky, 2020).

El apoyo de la maestra a los niños durante la resolución de los retos o problemas planteados desempeña un papel primordial para la superación de los obstáculos o los errores con los que se van encontrando a lo largo de la trayectoria de resolución, la comunicación y la colaboración juegan un papel imprescindible en la resolución de problemas (Sullivan y Bers, 2018; Wang et al., 2020). La intervención de la maestra ha cumplido unas premisas fundamentales para no condicionar las decisiones tomadas por el alumnado:

a) No apoya verbalmente para condicionar una respuesta determinada.

b) No corrige una respuesta errónea. La propuesta está diseñada para que sean los propios alumnos/as los que descubran sus errores y puedan reconducir la situación. Según Pólya (1945), los estudiantes practican la supervisión, considerada la última fase en la resolución de problemas matemáticos, mediante la cual, de manera autónoma, comprueban los planes ejecutados.

c) No interrumpe las verbalizaciones de los alumnos ni interviene en ellas.

d) Sí estimula a seguir con la resolución de problemas, animando al final de cada decisión para que sigan y confíen en sus decisiones.

e) Sí interviene ayudando únicamente en caso de bloqueo por parte de los alumnos. En las ocasiones en las que los estudiantes permanezcan un tiempo sin responder (un minuto o más) y gesticulan sin encontrar respuesta, la investigadora por medio de preguntas, ayuda al análisis de la situación por parte de los alumnos y a seguir con la resolución.

f) Nunca da la respuesta al problema planteado, sino que prepara a modo de andamiaje la trayectoria a seguir para su resolución.

g) Un problema se da por no solucionado si hay tres intentos fallidos o el alumnado tarda más de cinco minutos en comenzar el último intento de resolución. En ese caso se pasará al siguiente problema de la trayectoria de resolución de problemas.

La maestra, dicho lo anterior, interviene estrictamente para animar y fomentar la respuesta al problema planteado, en ningún momento su intervención condiciona las resoluciones (véase figura 2 ). 
Figura 2. Esquema de la intervención de la maestra

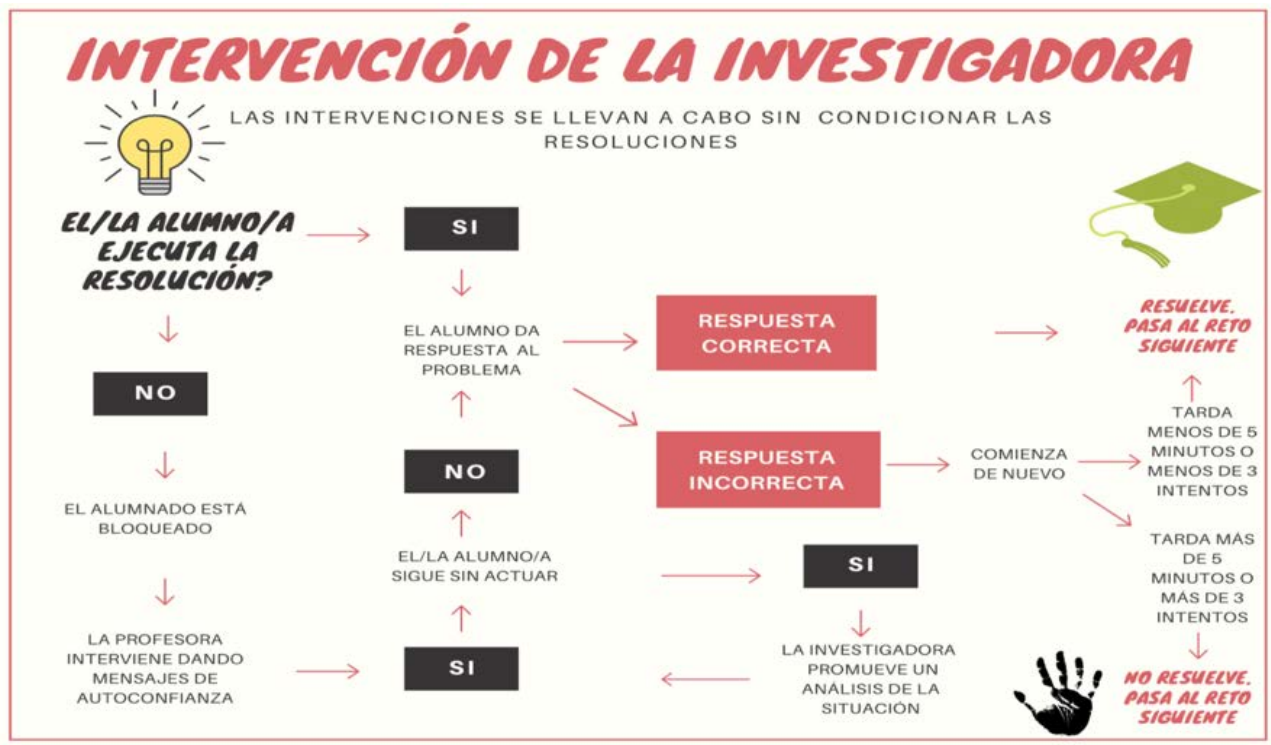

\subsection{Análisis de los datos}

Con el fin de establecer la existencia de asociación entre el criterio edad y el criterio acierto en cada step, se ha recurrido a la prueba $x^{2}$. Una vez determinada la asociación entre el grupo de edad y el cumplimiento o no de un determinado step, el interés se centra en analizar la dirección de la asociación, positiva o negativa, a través del análisis de los residuos ajustados; este análisis contrastará entre qué edades hay más posibilidad de acertar o errar un determinado step (Haberman, 1973).

Por otro lado, para determinar si existen diferencias estadísticamente significativas entre los grupos de edad en lo relativo al tiempo empleado para la resolución del conjunto de los steps, así como en cada uno de ellos, se recurre a la prueba no paramétrica de Kruskal Wallis, ya que la selección de la muestra no se ha realizado aleatoriamente. En el caso de concluirse que el tiempo empleado en al menos uno de los grupos es significativamente diferente de los demás, se procede a realizar un contraste entre pares de grupos a través de la prueba $U$ de Mann-Whitney, para constatar entre qué parejas hay diferencias y el sentido de esta diferencia (Carver y Nash, 2006).

\section{Resultados}

\subsection{Resultados de las resoluciones ofrecidas por cursos}

A continuación, se presenta en la tabla 5, la proporción de participantes por grupo de edad que cumplimentan con éxito cada uno de los steps que constituyen la trayectoria de problemas. 
Tabla 5. Proporción de participantes por grupo edad que cumplimentan cada uno de los steps de la trayectoria de resolución de problemas.

\begin{tabular}{|c|c|c|c|}
\hline Step & $1^{\circ} \mathrm{EI}$ & $2^{\circ} \mathrm{EI}$ & $3^{\circ} \mathrm{El}$ \\
\hline $\mathrm{S} 1$ & $12 / 12$ & $11 / 11$ & $11 / 11$ \\
\hline S2 & $10 / 12$ & $11 / 11$ & $11 / 11$ \\
\hline S3 & $8 / 12$ & $9 / 11$ & $11 / 11$ \\
\hline S4 & $6 / 12$ & $9 / 11$ & $11 / 11$ \\
\hline S5 & $5 / 12$ & $11 / 11$ & $10 / 11$ \\
\hline S6 & $9 / 12$ & $11 / 11$ & $11 / 11$ \\
\hline S7 & $1 / 12$ & $6 / 11$ & $4 / 11$ \\
\hline S8 & $7 / 12$ & $9 / 11$ & $11 / 11$ \\
\hline
\end{tabular}

Un análisis global, a través de la prueba $x^{2}$ de Pearson, muestra que existe asociación entre el criterio grupo de edad y los criterios: step $4\left(x^{2}=8.233 ; \mathrm{gl}=2 ; p=.016\right)$; step $5\left(x^{2}=11.066 ; \mathrm{gl}=2 ; p=.004\right) ;$ y step $6\left(x^{2}=6.032 ; g \mathrm{l}=2 ; p=.049\right)$. En concreto, un análisis de residuos corregidos (Haberman, 1973), indica que los participantes de $1^{\circ}$ de Educación Infantil es probable que no acierten el step $4(\mathrm{r}=2.7)$, mientras los participantes de $3^{\circ}$ de Educación Infantil es probable que lo superen $(r=2.2)$; que los participantes de $1^{\circ}$ de Educación Infantil es probable que no acierten el step $5(r=3.2)$, mientras que el grupo de $2^{\circ}$ de Educación Infantil es probable que lo superen $(r=2.4)$; y que los participantes de $1^{\circ}$ de Educación Infantil es probable que no superen el step $6(r=2.5)$.

\subsection{Resultados relativos al tiempo empleado en la resolución por cursos}

En la tabla 6 se presentan la media y desviación típica de los tiempos empleados por cada grupo de edad en la resolución de los ocho steps constitutivos de la trayectoria de resolución de problemas.

Tabla 6. Media y desviación típica de los tiempos, en segundos, empleados por cada grupo de edad en la resolución de los ocho steps constitutivos de la trayectoria de resolución de problemas

\begin{tabular}{ccccccc}
\hline Step & \multicolumn{2}{c}{$\mathbf{1}^{\mathbf{o}}$ Educación Infantil } & \multicolumn{2}{c}{$\mathbf{2}^{\mathbf{o}}$ Educación Infantil } & \multicolumn{2}{c}{$\mathbf{3}^{\mathbf{o}}$ Educación Infantil } \\
& Media & Desv. Tip & Media & Desv. Tip & Media & Desv. Tip \\
\hline S1 & 241.333 & 77.759 & 175.636 & 56.759 & 117.454 & 17.823 \\
\hline S2 & 344.083 & 74.266 & 233.909 & 75.047 & 190.818 & 64.204 \\
\hline S3 & 314.083 & 85.361 & 244.090 & 104.544 & 228.363 & 94.398 \\
\hline S4 & 400.916 & 114.618 & 257.727 & 45.387 & 244 & 73.441 \\
\hline S5 & 388.583 & 91.555 & 261.181 & 111.054 & 290.272 & 74.241 \\
\hline S6 & 280.083 & 110.803 & 198.909 & 67.423 & 188.090 & 60.535 \\
\hline
\end{tabular}




\begin{tabular}{ccccccc}
\hline \multirow{2}{*}{ Step } & \multicolumn{2}{c}{$\mathbf{1}^{\mathbf{o}}$ Educación Infantil } & \multicolumn{2}{c}{$\mathbf{2}^{\mathbf{o}}$ Educación Infantil } & \multicolumn{2}{c}{$\mathbf{3}^{\mathbf{o}}$ Educación Infantil } \\
& Media & Desv. Tip & Media & Desv. Tip & Media & Desv. Tip \\
\hline S7 & 446.916 & 83.191 & 420.545 & 65.289 & 449 & 51.095 \\
\hline S8 & 223.083 & 105.943 & 158.636 & 95.078 & 105.727 & 28.485 \\
\hline
\end{tabular}

Para determinar si existen diferencias significativas entre los grupos de edad en el tiempo empleado para la resolución del conjunto de los steps, se recurre a la prueba no paramétrica de Kruskal Wallis. El resultado $\left(x^{2}=39.77 ; p<.001\right)$ señala que el tiempo empleado en al menos uno de los grupos es significativamente diferente de los demás. Para determinar, entre que pares de grupos hay diferencias significativas se ha realizado la prueba $U$ de Mann-Whitney; se constata que los participantes de $1^{\circ}$ de Educación Infantil emplean más tiempo en la resolución de los steps que los integrantes de $2^{\circ}$ de Educación Infantil (U de Mann-Whitney $=2439.50 ; p<.001$ ) y de $3^{\circ}$ de Educación Infantil ( $U$ de Mann-Whitney=2166.50; $p<.001$ ); mientras que no se encuentran diferencias significativas entre los componentes de $2^{\circ}$ de Educación Infantil y $3^{\circ}$ de Educación Infantil (U de Mann-Whitney=3396.50; $p=.159$ ).

Si aplicamos la prueba no paramétrica de Kruskal Wallis al conjunto de los grupos de edad y en cada uno de los steps, comprobamos que existen diferencias estadísticamente significativas en el tiempo empleado para la cumplimentación por step: step $1\left(X^{2}=19.160 ; \mathrm{gl}=2 ; p<.001\right)$, step $2\left(x^{2}=16.037 ; \mathrm{gl}=2 ; p<.001\right)$, step $4\left(x^{2}=15.842\right.$; $\mathrm{gl}=2 ; p<.001)$, step $5\left(x^{2}=8.927 ; \mathrm{gl}=2 ; p<.001\right)$, step $6\left(x^{2}=6.288 ; \mathrm{gl}=2 ; p<.001\right) \mathrm{y}$ step $8\left(x^{2}=7.650 ; g \mid=2 ; p<.001\right)$. Profundizando en el análisis par a par entre grupos de edad, se ha constatado mediante la prueba $U$ de Mann-Whitney las diferencias estadísticamente significativas que se recogen en la tabla 7.

Tabla 7. Diferencias estadísticamente significativas en el análisis par a par entre grupos de edad y por step

\begin{tabular}{|c|c|c|c|c|c|c|}
\hline \multirow[t]{2}{*}{ Step } & \multicolumn{2}{|c|}{$1^{\circ} \mathrm{EI}-2^{\circ} \mathrm{EI}$} & \multicolumn{2}{|c|}{$1^{\circ} \mathrm{El}-3^{\circ} \mathrm{EI}$} & \multicolumn{2}{|c|}{$2^{\circ} \mathrm{EI}-3^{\circ} \mathrm{EI}$} \\
\hline & $\begin{array}{c}\text { U de Mann- } \\
\text { Whitney }\end{array}$ & Significación & $\begin{array}{l}\text { U de Mann- } \\
\text { Whitney }\end{array}$ & Significación & $\begin{array}{c}\text { U de Mann- } \\
\text { Whitney }\end{array}$ & Significación \\
\hline S1 & 33.000 & .042 & 2 & .000 & 14.500 & .003 \\
\hline $\mathrm{S} 2$ & 19.000 & .004 & 7 & .000 & & \\
\hline S3 & 33.000 & .042 & 36.5 & & & \\
\hline S4 & 12.000 & .001 & 11 & 0.001 & & \\
\hline S5 & 23.000 & .008 & 27.5 & 0.018 & & \\
\hline S6 & & & 27.5 & 0.018 & & \\
\hline S7 & & & 64 & & & \\
\hline S8 & & & 21 & 0.006 & & \\
\hline
\end{tabular}

\section{Discusión y CONCLUSión}

El pensamiento computacional se sitúa en el eje de la innovación educativa y la enseñanza de disciplinas como la robótica y el pensamiento computacional se perfila como experiencias cautivadoras en Educación Infantil (Bers et al., 2019; Román- 
González et al., 2015). Con la intención de desarrollar las habilidades de pensamiento computacional en el alumnado de Educación Infantil se ha desarrollado la propuesta de trayectoria de resolución de problemas para el desarrollo del pensamiento computacional mediante el empleo de un robot de suelo de direccionalidad programada.

El diseño de propuestas de enseñanza fundamentadas en el uso de robots programables para trabajar la resolución de problemas tiene un enorme potencial para el desarrollo del pensamiento computacional en los escolares, permite la creación de un plan de intervención y la valoración del mismo (Diago et al., 2018b). La trayectoria de resolución de problemas planteada ha presentado un recorrido de resolución de dificultad creciente, posible de realizar, para que los estudiantes prosperen en su aprendizaje (Simon, 1995). Los retos planteados han ido haciéndose cada vez más complejos de resolver, comenzando por problemas con camino marcado y solución única: de un único giro (problemas 1 y 2), de doble giro (problemas 3 y 4), problemas sin recorrido marcado y con más de una solución (problemas 5, 6 y 7) y problema cuya solución era averiguar que no existe solución posible. Los escolares que han participado en la propuesta han sido capaces de aplicar los conocimientos que han ido adquiriendo a lo largo de la puesta en práctica de la trayectoria de resolución de problemas para tratar de resolver los retos más complicados. Dichos retos han implementado la solución con un dispositivo tecnológico, el robot Next 1.0, que ha sido el encargado de realizar el trayecto planificado por los escolares y gracias al cual se ha podido efectuar una evolución del mismo en función de las respuestas ofrecidas por el robot. Los problemas de la trayectoria de resolución han proporcionado información relativa al modo de comprender y resolver problemas de los escolares de Educación Infantil. Los estudiantes han sido capaces de descomponer los problemas en partes más sencillas, seleccionando para ello las tarjetas necesarias para que Next fuera avanzando; además han asimilado los patrones de movimiento en función de las tarjetas y el movimiento del robot, haciendo uso de la codificación para resolver de manera lógica los retos planteados.

La secuencia programada de problemas englobados en una trayectoria de resolución de problemas de dificultad creciente permite a la maestra desarrollar el talento matemático ya que potencia la creatividad en las respuestas dadas por el alumnado (Butto et al., 2016; Taylor, 1964). Los retos de solución abierta (problemas 5, 6, 7 y 8), más complejos en cuanto a resolución, han permitido desarrollar el potencial del alumnado con talento matemático, ya que estos escolares han podido mostrar su curiosidad por los problemas más complicados, han podido descubrir la clave de los problemas con mayor facilidad que el resto de los compañeros, han podido dar respuestas con soluciones alternativas, como en el problema cuya solución era concluir que no tenía solución y han podido mostrar una gran implicación en las tareas, lo que ha conllevado a poder alcanzar un mayor rendimiento en la experimentación (Freiman, 2006). Sírvase de ejemplo de respuesta de un alumno de $1^{\circ}$ de Educación Infantil (edad: 3 años, 5 meses y 19 días), que a los 26 segundos de haberle enunciado el problema descubre que "no tiene paso Next" y, sin pedirle una solución alternativa, ofrece una respuesta original alternativa consistente en quitar a uno de los ogros que bloquean el camino a los 56 segundos, la ejecuta y resuelve, dando una solución innovadora, pudiendo ser un indicador de alta capacidad matemática (Castro et al., 2006). La capacidad mostrada por dicho estudiante para generar ideas creativas, es 
un indicador del talento matemático (Reyes-Santander y Karg, 2009). Varios son los estudiantes que han demostrado perseverancia en la tarea, involucrándose en la resolución de la trayectoria e incluso generando respuestas creativas, especialmente en el problema sin solución, mostrando características indicadoras de talento matemático. La resolución de problemas matemáticos, en este caso llevada a cabo por medio del pensamiento computacional, es un gran medio para distinguir a los escolares con talento matemático (Castro et al., 2006); además, como señalan Reyes-Santander y Karg (2009), mediante la puesta en práctica de tareas como las descritas, se brinda al profesorado un escenario excepcional para identificar el talento matemático.

El segundo objetivo propuesto pretende analizar la adecuación de la propuesta de resolución de problemas al pensamiento computacional del alumnado de los tres cursos del segundo ciclo de Educación Infantil, a partir de las resoluciones ofrecidas y del tiempo empleado en la resolución de cada uno de los problemas que componen la trayectoria. El estudio de los resultados obtenidos para cada uno de los cursos muestra cómo, a medida que se avanza en edad, los escolares son más capaces de realizar resoluciones correctas a los problemas planteados y necesitan menos tiempo para resolver los problemas, lo que aporta información sobre la progresiva adquisición de las habilidades de resolución inducidas por la comprensión del procedimiento de resolución en Educación Infantil. La integración de la robótica y la programación en las aulas de Educación Infantil provoca un impacto en las habilidades de secuenciación, proceso fundamental del pensamiento computacional (Kazakoff y Bers, 2011; Kazakoff et al., 2013; Wing, 2006).

Para concluir destacar cómo la propuesta de experimentación desarrollada ha permitido analizar el razonamiento matemático de los escolares de Educación Infantil. Además, ha posibilitado realizar un análisis del pensamiento computacional del alumnado mediante la trayectoria de resolución de problemas mostrando diferencias en las habilidades de resolución en función de la edad en los procesos involucrados. Actividades intelectuales innovadoras como las descritas proporcionan retos desafiantes y muy interesantes para el alumnado de Educación Infantil creando un entorno de libertad para expresar respuestas originales y brindan un escenario único para potenciar a los estudiantes con alta capacidad matemática.

\section{REFERENCIAS BIBLIOGRÁFICAS}

Benton, L., Hoyles, C., Kalas, I. y Noss, R. (2017). Bridging primary programming and mathematics: Some findings of design research in England. Digital Experiences in Mathematics Education, 3(2), 115-138. https://doi.org/10.1007/s40751-0170028-x

Bers, M. (2018). Coding as a Playground: Programming and Computational Thinking in the Early Childhood Classroom. Routledge.

Bers, M., González, C. y Armas, U. (2019). Coding as a playground: Promoting positive learning experiences in childhood classrooms. Computers \& Education, 138, 130-145. https://doi.org/10.1016/j.compedu.2019.04.013

Butto, C., Andrade A. y Lanz M. Y. (2016). Identificación de estudiantes con altas capacidades matemáticas en educación primaria. Horizontes Pedagógicos, 18(2), 66-85. 
Carver, R. H. y Nash, J. G. (2006). Doing data analysis with SPSS. Boston: Brooks/ Cole.

Castro, E., Benavides, M. y Segovia, I. (2006). Cuestionario para caracterizar a niños con talento en resolución de problemas de estructura multiplicativa. Faisca: revista de altas capacidades, 11(13), 4-22.

Clements, D. H. y Samara, J. (2002). The Role of Technology in Early Childhood Learning. Teaching Children Mathematics, 8(6), 340-343.

Denis, M. (2017). Space and spatial cognition: A multidisciplinary perspective. Routledge.

Diago, P. D., Arnau, D., y González-Calero, J. A. (2018a). Elementos de resolución de problemas en primeras edades escolares con Bee-bot. Edma 0-6: Educación matemática en la infancia, 7(1), 12-41.

Diago, P. D., Arnau, D. y González-Calero, J. A. (2018b). La resolución de problemas matemáticos en primeras edades escolares con Bee-bot. Matemáticas, Educación y Sociedad, 1(2), 36-50.

Etchepare, G. C., Ortega, R., Pérez, C., Flores, C. y Melipillán, R. (2011). Inteligencia lógica y rendimiento académico en matemáticas: un estudio con estudiantes de Educación Básica y Secundaria de Chile. Anales de Psicología, 27(2), 389-398.

Ferrándiz, C., Prieto, D., Fernández, C., Soto, G., Ferrando, M. y Badía, M. (2010). Modelo de identificación de alumnos con altas habilidades de educación secundaria. Revista Electrónica Interuniversitaria de Formación del Profesorado, 13(1), 63-74.

Freiman, V. (2006). Problems to discover and boost mathematical talent in early grades: A challenging situations approach. The Mathematics Enthusiast, 3(1), 51-75.

Gouws, L. A., Bradshaw, K., y Wentworth, P. (2013). Computational thinking in educational activities: An evaluation of the educational game light-bot. En Proceedings of the 18th ACM Conference on Innovation and Technology in Computer Science Education, Canterbury, United Kingdom, 10-15.

Greenes, C. (1981). Identifying the Gifted Student in Mathematics. The Arithmetic Teacher, 28(6), 14-17.

Haberman, S. J. (1973). The Analysis of Residuals in Cross-Classified Tables. Biometrics, 29, 205-220.

Hoyles, C. y Lagrange, J. B. (Eds.). (2010). Mathematics Education and TechnologyRethinking the Terrain: The 17th ICMI Study. New York: Springer.

Johnson, D. T. (2000). Teaching mathematics to gifted students in a mixed-ability classroom. ERIC Clearinghouse on Disabilities and Gifted Education.

Kazakoff, E. y Bers, M. (2011, April). The impact of computer programming on sequencing ability in early childhood. In American Educational Research Association Conference (AERA), Louisiana: New Orleans.

Kazakoff, E., Sullivan, A. y Bers, M. (2013). The effect of a classroom-based intensive robotics and programming workshop on sequencing ability in early childhood. Early Childhood Education Journal, 41(4), 245-255. https://doi.org/10.1007/ s10643-012-0554-5 
Kewalramani, S., Palaiologou, I., Arnott, L. y Dardanou. M. (2020). The integration of the internet of toys in early childhood education: a platform for multi-layered interactions, European Early Childhood Education Research Journal, 28(2), 197-213. https://doi.org/10.1080/1350293X.2020.1735738

Krutetskii, V. A. (1976). The psychology of mathematical abilities in school children. Chicago: The University of Chicago Press.

Lee, I., Martin, F., Denner, J., Coulter, B., Allan, W., Erickson, J., Malyn-Smith, J. y Werner, L. (2011). Computational thinking for youth in practice. Acm Inroads, 2(1), 32-37.

Leidl, K., Bers, M. U. y Mihm, C. (2017). Programming with ScratchJr: a review of the first year of user analytics. In International Conference on Computational Thinking Education, 2017. Wanchai, Hong Kong.

Manches, A. y Plowman, L. (2017). Computing education in children's early years: A call for debate. British Journal of Educational Technology, 48(1), 191-201. https:// doi.org/10.1111/bjet.12355

Moreno-León, J. y Robles, G. (2015, March). The Europe Code Week (CodeEU) initiative shaping the skills of future engineers. En 2015 IEEE Global Engineering Education Conference (EDUCON), Tallin, Estonia (pp. 561-566). https://doi. org/10.1109/EDUCON.2015.7096025

Onrubia, J., Rochera, M. y Barberá, E. (2003). La Enseñanza y el Aprendizaje de las Matemáticas: Una Perspectiva Psicológica. En J. Palacios, A. Marchesi y C. Coll (Eds.), Desarrollo Psicológico y Educación 1. Psicología Evolutiva (pp. 453-469). Madrid: Alianza.

Özdemir, D. A. e Işiksal, M. (2019). Mathematically gifted students' differentiated needs: what kind of support do they need? International Journal of Mathematical Education in Science and Technology, 1-19. https://doi.org/10.1080/002073 9X.2019.1658817

Pérez, G. y Diago, P. D. (2018). Estudio exploratorio sobre lenguajes simbólicos de programación en tareas de resolución de problemas con Bee-bot. Magister: Revista de Formación del Profesorado e Investigación Educativa, 30 (1 y 2), 9-20. https:// doi.org/10.17811/msg.30.1.2018.9-20

Piñeiro, J., Ramírez-Uclés, R. y Segovia-Álex, I. (2018). Detección del talento matemático en Educación Infantil. Edma 0-6: Educación matemática en la infancia, 6(2), 56-71

Pitta-Pantazi, D. P., Christou, C., Kontoyianni, K. y Kattou, M. (2011). A model of mathematical giftedness: Integrating natural, creative, and mathematical abilities. Canadian Journal of Science, Mathematics and Technology Education, 11(1), 3954.

Pólya, G. (1945). How to solve it. Princeton, New Jersey: Princeton University.

Pöntinen, S. y Räty-Záborszky, S. (2020). Pedagogical aspects to support students' evolving digital competence at school. European Early Childhood Education Research Journal, 28(2), 182-196. https://doi.org/10.1080/1350293X.2020.1735736

Ribera, J. M. (2021). Estrategias para la resolución de problemas de matemáticas a través del pensamiento computacional. En F. Navaridas y E. Raya (Eds.), Formación 
docente y desarrollo profesional del profesorado: Hacia un modelo para la calidad educativa. Madrid: Wolters Kluwer.

Román-González, M., Pérez-González, J. C. y Jiménez-Fernández, C. (14-16 de octubre de 2015). Test de Pensamiento Computacional: diseño y psicometría general. En III Congreso Internacional sobre Aprendizaje, Innovación y Competitividad, Madrid, España (pp. 1-6). http://doi.org/10.13140/RG.2.1.3056.5521

Shute, V. J., Sun, C. y Asbell-Clarke, J. (2017). Demystifying computational thinking. Educational Research Review, 22, 142-158. http://doi.org/10.1016/j.edurev.2017.09.003

Simon, M. A. (1995). Reconstructing mathematics pedagogy from a constructivist perspective. Journal for Research in Mathematics Education, 26(2), 114-145.

Skoumpourdi, C. (2010). Kindergarten mathematics with 'Pepe the Rabbit': how kindergartners use auxiliary means to solve problems. European Early Childhood Education Research Journal, 18(3), 299-307. http://dx.doi.org/10.1080/135029 $3 X .2010 .500070$

Sullivan, A. y Bers, M. (2016). Robotics in the early childhood classroom: learning outcomes from an 8-week robotics curriculum in pre-kindergarten through second grade. International Journal of Technology and Design Education, 26(1), 3-20. http://doi.org/10.1007/s10798-015-9304-5

Szendrei, J. (1996). Concrete Materials in the Classroom. En Bishop A.J., Clements K., Keitel C., Kilpatrick J., Laborde C. (eds), International Handbook of Mathematics Education vol. 4 (pp. 411-434). Dordrecht: Springer. https://doi.org/10.1007/97894-009-1465-0_12

Taylor, C. (1964): Creativity: Progress and potential. New York: Mc Graw-Hill.

Terroba, M., Ribera, J. M. y Lapresa, D. (2020). Pensamiento computacional en la resolución de problemas contextualizados en un cuento en Educación Infantil. Edma 0-6: Educación matemática en la infancia, 9(2), 73-92.

Valverde, J., Fernández, M. R., y Garrido, M. C. (2015). El pensamiento computacional y las nuevas ecologías del aprendizaje. RED-Revista de educación a distancia, 46(3). https://doi.org/10.6018/red/46/3

Wang, X. C., Choi, Y., Benson, K., Eggleston, C., y Weber, D. (2020). Teacher's role in fostering preschoolers' computational thinking: An exploratory case study. Early Education and Development, 1-23. https://doi.org/10.1080/10409289.2020.175 9012

Wing, J. M. (2006). Computational Thinking. Communications of the ACM, 49(3), 3335. http://doi.org/10.1145/1118178.1118215 